\section{FRI0724 PREDICTORS OF PRESENTEEISM AND ABSENTEEISM IN PATIENTS COMMENCING TREATMENT WITH METHOTREXATE MONOTHERAPY OR BIOLOGIC THERAPY FOR RHEUMATOID ARTHRITIS}

S. Leggett ${ }^{1}$, M. Lunt ${ }^{1}$, A. Barton ${ }^{2}$, K. Hyrich ${ }^{1}$, K. Walker-Bone ${ }^{3}$, S.M. Verstappen ${ }^{1}$ on behalf of RAMS and BRAGGSS co-investigators. ${ }^{1}$ Arthritis Research UK Centre for Epidemiology; ${ }^{2}$ Arthritis Research UK Centre for Genetics and Genomics, The University of Manchester, Manchester; ${ }^{3}$ Arthritis Research UK/MRC Centre for Musculoskeletal Health and Work, The University of Southampton, Southampton, United Kingdom

Background: The adverse effects of RA on absenteeism (i.e. days absent from work) and presenteeism (at-work productivity loss) are increasingly recognised as an important issue. Research suggests the reasons are multifactorial with many factors such as disease severity, quality of life, and depression possibly contributing to poor work outcomes in patients with RA.

Objectives: To identify predictors of presenteeism and absenteeism over one year in patients with RA commencing treatment with methotrexate (MTX) or biologics for the first time.

Methods: Patients recruited to the Rheumatoid Arthritis Methotrexate Starters study (RAMS) or the Biologics in Rheumatoid Arthritis Genetics and Genomics Study Syndicate (BRAGGSS), and in full or part-time paid employment were included in this analysis. Demographic (e.g., age), clinical (e.g., DAS28), and psychological data (e.g., Hospital Anxiety and Depression scale (HADS)) were collected at baseline. Patients were followed up at six months and one year in both cohorts. Absenteeism in the last month (days missed), and presenteeism ( 0 $=$ no interference $-10=$ complete interference), were measured at all three points using the RA specific Work Productivity Survey (WPS-RA). Patients with at least one follow-up post baseline were included in this analysis. Due to excessive zeros in the WPS-RA, a repeated measure zero inflated negative binomial regression (ZINB) was used to test the association between baseline demographic, clinical and psychological variables and presenteeism and absenteeism over one year. Results: Data was available for 191 patients in BRAGGSS and 308 in RAMS. In BRAGGSS, the mean age was 51 years (SD 9.5), median symptom duration was 8 years (IQR $5-13$ ); $78 \%$ female. In RAMS, the mean age was 52 years (SD 9.6), median symptom duration was 8.5 months (IQR 5-23); 66\% female. At baseline, $26 \%$ (BRAGGSS) and $27 \%$ (RAMS) reported $\geq 1$ days absent from work, and the median presenteeism scores were 5 (IQR 3-7) and 4 (IQR 1-7) for BRAGGSS and RAMS respectively. Presenteeism scores significantly improved over 1 year in both cohorts (BRAGGSS: median 2 [IQR 0-5], RAMS: median 2 (IQR $0-4)$ Kruskal-Wallis $p<0.001)$ ), as did absenteeism for both cohorts although non-significant. The ZINB revealed similar predictive patterns in both cohorts for presenteeism and absenteeism (Table 1). For example, for every 0.06 increase in EQ-5D (clinically important difference), the odds of being in the zero presenteeism group (i.e., no at-work productivity loss) over one year increased by a factor of 1.2 in BRAGGSS, and was similar in RAMS. In RAMS, a one unit increase in VAS fatigue decreased the odds of having no absenteeism over one year by a factor of 0.97 .

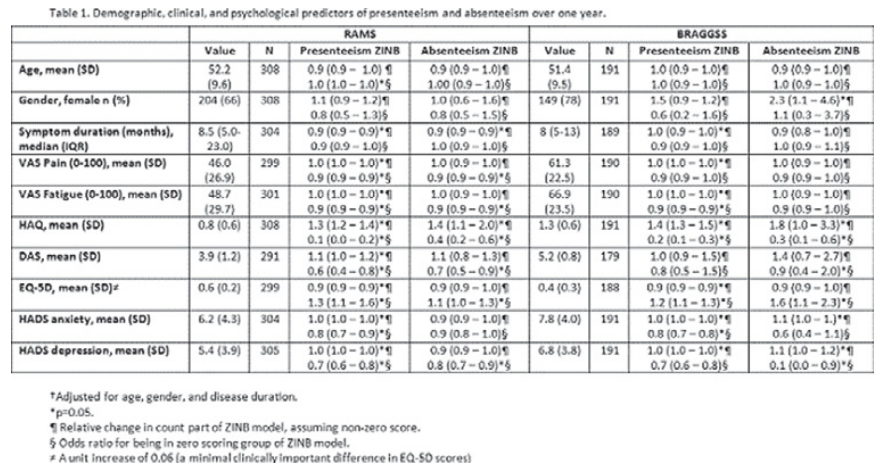

Conclusions: The results from this analysis support the notion of a multifactorial relationship between RA and work outcomes. The results suggest that greater quality of life and psychological well-being, amongst other factors, can predict improvements in presenteeism and absenteeism at one year in patients on either MTX or biologics for RA.

Acknowledgements: Funding: Pfzier I-CRP

Disclosure of Interest: None declared

DOI: 10.1136/annrheumdis-2017-eular.5077

\section{FRI0725 PREDICTION OF INTERSTITIAL LUNG DISEASE IN MIXED CONNECTIVE TISSUE DISEASE}

S. Reiseter ${ }^{1}$, R. Gunnarsson ${ }^{2}$, T.M. Aaløkken ${ }^{3}$, M.B. Lund ${ }^{4}$, J. Corander ${ }^{5}$, Ø. Molberg ${ }^{6} .{ }^{1}$ Institute of Clinical Medicine, University of Oslo; ${ }^{2}$ Dept. of Rheumatology: ${ }^{3}$ Dept. of Radiology and Nuclear Medicine; ${ }^{4}$ Dept. of Respiratory Medicine, Oslo University Hospital; ${ }^{5}$ Institute of Basic Medical Sciences; ${ }^{6}$ Institute of Clinical Medicine, University of Oslo, Oslo, Norway

Background: Mixed Connective Tissue Disease (MCTD) is characterized by high levels of autoantibodies against U1 small nuclear ribonucleoprotein (RNP) and clinical manifestations also found in Systemic Sclerosis, Systemic Lupus Erythematosus, Rheumatoid Arthritis and Polymyositis. Interstitial Lung Disease (ILD) is a severe complication in MCTD and has been reported to affect between $35 \%$ and $85 \%$ (1) in different cohorts.

Objectives: Here we present a predicting model of ILD in an unselected nationwide MCTD cohort aiming to assist clinicians in identifying MCTD patients with ILD.

Methods: 135 patients with high resolution computed tomography (CT) available for systematic evaluation were included from our nationwide MCTD cohort. Abnormal CT findings of ground glass attenuation and reticular patterns were defined as ILD. Pulmonary function tests were performed within 2 months of the HRCT examination. Serum levels of anti-RNP and Ro-52 autoantibody were determined by line immunoassay (ANA Profile 5 Euroline Blot test kit, Euroimmun, Lübeck, Germany). Logistic regression analyses were used to find the predictive factors of ILD. Variables at a significant level of $P<0.25$ where considered a candidate in the prediction model by manual backward elimination procedure in addition to age and gender.

Results: Results: 52 patients (38\%) had evidence of ILD on HRCT. The predictive model is shown in Table 1. $P=0.55$ for goodness of fit test and $P=0.80$ for area under ROC curve.

\begin{tabular}{lccccccc}
\hline & \multicolumn{3}{c}{ Univariable } & & \multicolumn{3}{c}{ Multivariable } \\
\cline { 2 - 4 } \cline { 6 - 8 } & OR & $95 \% \mathrm{Cl}$ & $\mathrm{P}$ value & & $\mathrm{OR}$ & $95 \% \mathrm{Cl}$ & $\mathrm{P}$ value \\
\hline DLCO $<60$ & 5.8 & $2.3-14.6$ & $<.001$ & & 4.6 & $1.5-13.6$ & 0.006 \\
Anti-RNP antibodies titer $>200$ & 3.0 & $1.5-7.1$ & 0.003 & & 4.9 & $1.8-13.3$ & 0.003 \\
Never arthritis & 5.4 & $2.1-13.6$ & $<.001$ & & 3.3 & $1.1-9.9$ & 0.033 \\
Age group* at HRCT & 1.0 & $1.0-1.0$ & 0.015 & & 1.6 & $1.1-2.2$ & 0.012 \\
Anti-Ro52 antibodies present & 2.6 & $1.2-5.9$ & 0.021 & & & \\
FVC $<75$ & 3.0 & $1.1-8.2$ & 0.040 & & & \\
ESR $>30$ & 2.3 & $1.0-5.0$ & 0.043 & & & \\
Sclerodactily & 2.0 & $0.94-4.0$ & 0.071 & & & \\
Male gender & 1.6 & $0.71-3.5$ & 0.268 & & & \\
\hline
\end{tabular}

${ }^{*}$ Patients were divided in 6 age groups ( $<25$ years, 26 to 35 years, 36 to 45 years, 46 to 55 years, 56-65 years and above 65 years). FVC = Forced Vital Capacity \% of predicted, DLCO = diffusing capacity of the lung for carbon monoxide $\%$ of predicted, RNP = Ribonucleoprotein, ESR = erythrocyte sedimentation rate.

Conclusions: Risk factors of ILD in MCTD are DLCO less than $60 \%$ of predicted, high titer RNP antibodies, no previous arthritis and increasing age.

References:

[1] Gunnarsson R, Hetlevik SO, Lilleby V, Molberg O. Mixed connective tissue disease. Best Pract Res Clin Rheumatol. 2016;30(1):95-111.

Disclosure of Interest: None declared

DOI: 10.1136/annrheumdis-2017-eular.1841

\section{FRI0726 LONG TERM USE OF ANALGESICS AND NSAIDS IN EARLY RA: LESSONS FROM THE CARERA STUDY}

S. Pazmino $^{1}$, R. Westhovens ${ }^{1,2}$, V. Stouten ${ }^{1}$, J. Joly ${ }^{2}$, K. Van Der Elst ${ }^{2,3}$, P. Verschueren ${ }^{1,2}$ on behalf of CareRA study group. 'Skeletal Biology and Engineering Research Centre, KU Leuven Department of Development and Regeneration; ${ }^{2}$ Rheumatology, University Hospitals Leuven; ${ }^{3}$ Skeletal Biology and Engineering Research Centre, KU Leuven Department of Public Health and Primary Care, Leuven, Belgium

Background: One might consider pain accompanying musculoskeletal conditions as a separate illness entity deserving specific treatment. A subgroup of early Rheumatoid Arthritis (RA) patients has remaining pain despite adequate disease control and this might be reflected in the use of analgesics and NSAIDs.

Objectives: To investigate the usage of analgesics and antiphlogistics prospectively in the pragmatic randomized controlled CareRA trial and describe the users of such drugs taking into account body mass index (BMI), VAS pain and DAS28CRP.

Methods: This study utilized data from the CareRA trial, a 2-year prospective investigator-initiated multicentre pragmatic RCT in patients with early RA $(\leq 1$ year) comparing different early intensive treat to target strategies, including glucocorticoid (GC) bridging, aiming for remission in all participants.

All concomitant medication for each patient was recorded, including: name, dose, frequency/timing, continuous/intermittent use, start/end date and indication (possibly/definitely RA-related or other). Meaningful sub-classifications were made (analgesics, cox2-selective and non-selective NSAIDs). We defined two subgroups with prolonged analgesic/NSAID use till w104: the pre-induction group starting before/at baseline (BL) and the post-induction group starting w28-w52, after GC step-down.

Results: From the CareRA cohort $(n=379), 90 \%$ were on analgesics/NSAIDs at some time during the study and $84 \%$ had already started before inclusion (mean 24 weeks). In general, we observed a decreasing trend in the use of analgesics/NSAIDs, with a slight increase after GC step-down. Overall, 266 patients used non-selective and 131 cox2-selective NSAIDs, 154 paracetamol and 85 opioids.

Of the total population, $15,5 \%$ (53 patients) started long-term analgesics/NSAIDs before/at BL (pre-induction group) and 13\% (45 patients) from w28-w52 (postinduction group). 